\title{
Cycle Slip-Mitigating Turbo Demodulation in LDPC-Coded Coherent Optical Communications
}

Koike-Akino, T.; Kojima, K.; Millar, D.S.; Parsons, K.; Miyata, Y.; Matsumoto, W.; Sugihara, T.; Mizuochi, T.

TR2014-008 March 2014

\begin{abstract}
We show that an iterative demodulation with soft-decision feedback information from FEC decoder can efficiently mitigate cycle slips. With $3 \%$ pilot insertion, the turbo QPSK demodulation achieves $1.05 \mathrm{~dB}$ gain even in the presence of frequent cycle slips.
\end{abstract}

Optical Fiber Communication Conference and Exposition (OFC)

This work may not be copied or reproduced in whole or in part for any commercial purpose. Permission to copy in whole or in part without payment of fee is granted for nonprofit educational and research purposes provided that all such whole or partial copies include the following: a notice that such copying is by permission of Mitsubishi Electric Research Laboratories, Inc.; an acknowledgment of the authors and individual contributions to the work; and all applicable portions of the copyright notice. Copying, reproduction, or republishing for any other purpose shall require a license with payment of fee to Mitsubishi Electric Research Laboratories, Inc. All rights reserved. 



\title{
Cycle Slip-Mitigating Turbo Demodulation in LDPC-Coded Coherent Optical Communications
}

\author{
Toshiaki Koike-Akino ${ }^{1}$, Keisuke Kojima ${ }^{1}$, David S. Millar ${ }^{1}$, Kieran Parsons ${ }^{1}$, \\ Yoshikuni Miyata $^{2}$, Wataru Matsumoto ${ }^{2}$, Takashi Sugihara ${ }^{2}$, and Takashi Mizuochi ${ }^{2}$ \\ ${ }^{1}$ Mitsubishi Electric Research Laboratories (MERL), 201 Broadway, Cambridge, MA 02139, USA. \\ ${ }^{2}$ Information Technology R\&D Center, Mitsubishi Electric Corporation, 5-1-1, Ofuna, Kamakura, 247-8501, Japan \\ koike@merl.com
}

\begin{abstract}
We show that an iterative demodulation with soft-decision feedback information from FEC decoder can efficiently mitigate cycle slips. With $3 \%$ pilot insertion, the turbo QPSK demodulation achieves $1.05 \mathrm{~dB}$ gain even in the presence of frequent cycle slips.
\end{abstract}

OCIS codes: (060.4510) Optical communications, (060.1660) Coherent communications.

\section{Introduction}

In coherent optical communications, differentially-encoded quadrature phase-shift keying (DQPSK) has often been used for mitigating cycle slips caused by blind carrier-phase recovery (CPR). The cycle slip issue [1] is particularly important for forward error correction (FEC) coded systems because severe burst errors after cycle slips cannot be corrected [2]. Although differential encoding has a drawback of error propagation which can double bit-error rate (BER), DQPSK may be still better than QPSK in the presence of frequent cycle slips. Nevertheless, enormous effort to use QPSK rather than DQPSK has been conducted. For example, Zhang et al. [3] have reported a pilot-aided CPR. Wu et al. [4] have studied iterative CPR, in which soft-decision FEC output is fed back to CPR to correct phase estimation errors. Such a technique which collaborates between digital signal processing (DSP) and FEC decoder, a.k.a. a turbo principle [5-7], has drawn much attention for future advancement of optical communications [8] because recent FEC codes such as low-density parity-check (LDPC) codes have nearly achieved the Shannon limit [9].

In this paper, we investigate a turbo demodulation [10] as an alternative method of cycle slip mitigation. A turbo loop to exchange belief messages is formed between an FEC decoder and a demodulator. However, it has been reported that the turbo demodulation does not work well in the presence of cycle slips [11]. To overcome this problem, we introduce a trellis-based demodulator which uses a Markov model of cycle slips $[12,13]$ for robust belief calculations.

We use one of the recent LDPC codes [9] with $12 \mathrm{~dB}$ net coding gain (NCG) at a BER of $10^{-15}$. Since such a good FEC code has a long codeword (longer than 30000 bits), the impact of cycle slips is relatively large when the interleaver size is limited. We show that the turbo loop offers up to $1.4 \mathrm{~dB}$ gain, and our turbo demodulation of QPSK can outperform that of DQPSK by $0.6 \mathrm{~dB}$ for $3 \%$ pilot overhead even at a slip rate of above $10^{-3}$.

\section{Turbo demodulation robust to cycle slips}

We consider dual-polarization (DP) QPSK or DQPSK transmissions. A receiver DSP includes several pre-processing units that include resampling, timing recovery, deskew, polarization recovery, and chromatic dispersion compensation. Fig. 1 depicts the schematic of the digital post-processing unit. An automatic frequency controller (AFC) coarsely recovers the frequency offset. The residual carrier phase fluctuation is compensated by conventional Viterbi \& Viterbi (V\&V) CPR. Since such a blind CPR cannot perfectly lock the carrier phase, cycle slips can occur with a certain probability. It is known [12] that the slip occurrence can be modeled by Markov process, where phase state transition can be expressed by a transition probability matrix.

In order to detect the undesired cycle slips that have occurred at CPR, we adopt a maximum a posteriori probability (MAP) based demodulator which uses both scattered pilots and data through a trellis diagram in a Markov model. Considering possible slip state transitions in the Markov model, we can generate robust belief messages of log-likelihood ratio (LLR) for the following LDPC decoder. We use a max-log-MAP algorithm, which is a simplified version of MAP.

The LLR messages from the MAP demodulator are fed into variable-node decoders (VND) for LDPC decoding. With sum-product belief propagation algorithm, the belief messages are exchanged between VND and check-node decoders (CND). The iterative LDPC decoding has this inner-loop iteration to correct errors. After several inner-loop iterations, the soft-decision LLR messages of VND are taken by a hard decision unit to obtain decoded binary data. 


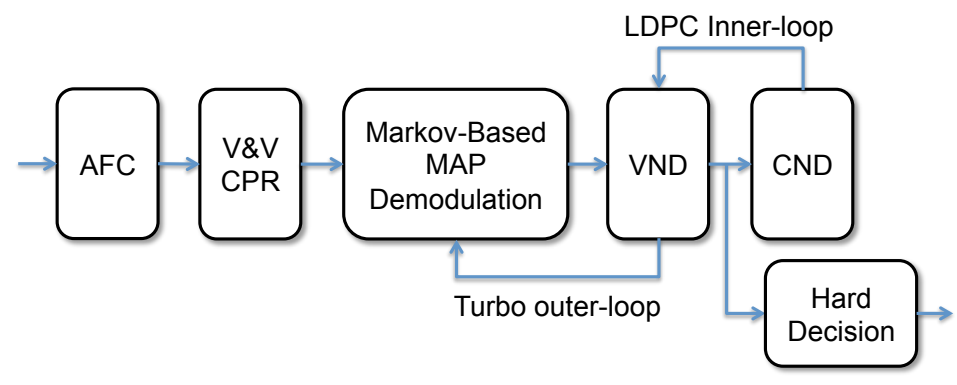

Fig. 1: Turbo demodulation for cycle-slip recovery.

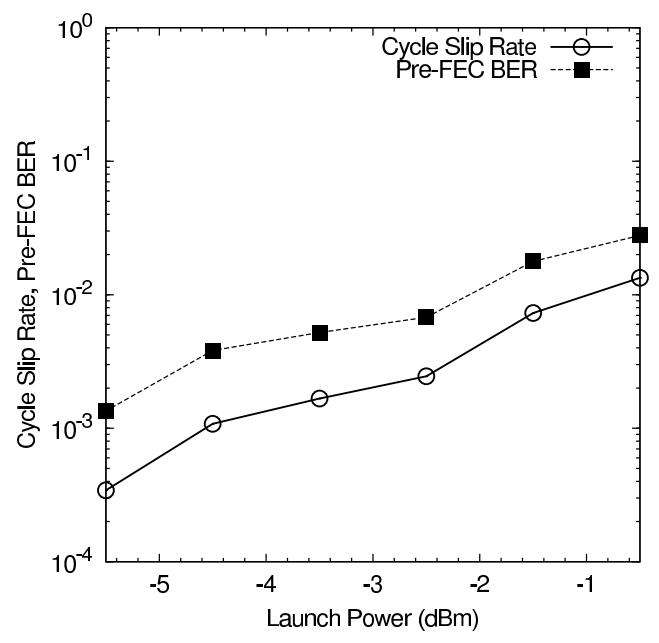

Fig. 2: Measured slip rate vs. launch power.

Before taking hard decisions, we can feed back the soft-decision information to the MAP demodulator. With the FEC feedback information, the MAP demodulator can improve the LLR information because cycle slips can be more accurately detected. This feedback loop from FEC decoder to MAP demodulator is called the turbo outer-loop. Our purpose is to show the impact of the turbo outer-loops for cycle slip mitigation, and to compare QPSK and DQPSK.

\section{Cycle slip rate measurement}

To observe the behavior of cycle slips, we made a laboratory measurement. The experimental fiber link configuration [14] is as follows. The channel is a 10.7 GBd non-return-to-zero DP-QPSK signal with a center wavelength of $1551.32 \mathrm{~nm}$. After pre-dispersion compensation, the signal is propagated through 5 loops of 18 spans of non-zero dispersion shift fiber (NZ-DSF) and 3 spans of standard single-mode fiber (SSMF) with compensating erbium-doped fiber amplifiers (EDFAs), post-dispersion compensation and an optical filter. The fiber distance per loop is $1,046 \mathrm{~km}$, and the total line distance is $5,230 \mathrm{~km}$. We use frequency-domain dispersion compensation, 7-tap constant-modulus algorithm (CMA) polarization recovery with least-mean-square (LMS) adaptation, and 19-tap V\&V CPR.

In Fig. 2, we plot the measured slip rate as a function of launch power. The slip rate is the ratio between the number of slip counts and the number of symbols. We also present the pre-FEC BER performance, which degrades with increased launch power due to nonlinear distortions. It can be seen that the cycle slip rate is relatively high (above $10^{-3}$ ) in FEC limit regimes (above $10^{-2}$ ), and more importantly the slip rate is no lower than one tenth of the pre-FEC BER. Note that the slip rate can be improved by other sophisticated CPRs [1]. This experimental result is used for modeling the slip rate in the following simulations for turbo demodulation of LDPC-coded $100 \mathrm{~Gb} / \mathrm{s}$ transmissions.

\section{Performance results of turbo demodulation}

We evaluate the post-FEC BER for $100 \mathrm{~Gb} / \mathrm{s}$ transmission through computer simulations in the presence of frequent cycle slips, whose rate is one tenth of pre-FEC BER. We use one of the world-best LDPC codes [9] with 12 dB NCG and a code length of 38400. This LDPC code is a class of capacity-approaching spatially-coupled irregular codes. In Fig. 3, we plot the post-FEC BER performance as a function of optical signal-to-noise power ratio (OSNR) at a reference bandwidth of $0.1 \mathrm{~nm}$ in back-to-back configuration. We assume $32.89 \sim 33.98 \mathrm{GBd}$ rate depending on pilot overhead. The total number of inner-loop iterations for LDPC decoding is 32, while no turbo outer-loop iteration is used as in a conventional DSP. To simulate the worst-case scenario, we do not use an interleaver such that burst errors directly degrade post-FEC BER. We also present the BER performance for an idealistic case with no cycle slips.

As shown in Fig. 3, the post-FEC BER significantly degrades in the presence of cycle slips. The performance loss from the ideal case is $2.75 \mathrm{~dB}, 1.9 \mathrm{~dB}$, and $1.35 \mathrm{~dB}$ for $1 \%, 2 \%$, and $3 \%$ pilot insertion, respectively, at a BER of $10^{-5}$ (it is a reasonable reference BER to predict performance even at lower BER in waterfall regimes). On the other hand, DQPSK shows almost no degradation caused by cycle slips. Although the ideal DQPSK itself exhibits a performance loss of $1.45 \mathrm{~dB}$ from ideal QPSK due to error propagation from differential encoding, the post-FEC BER of QPSK in the presence of cycle slips becomes worse than that of DQPSK for the case with low-overhead pilots (less than $3 \%$ ). 


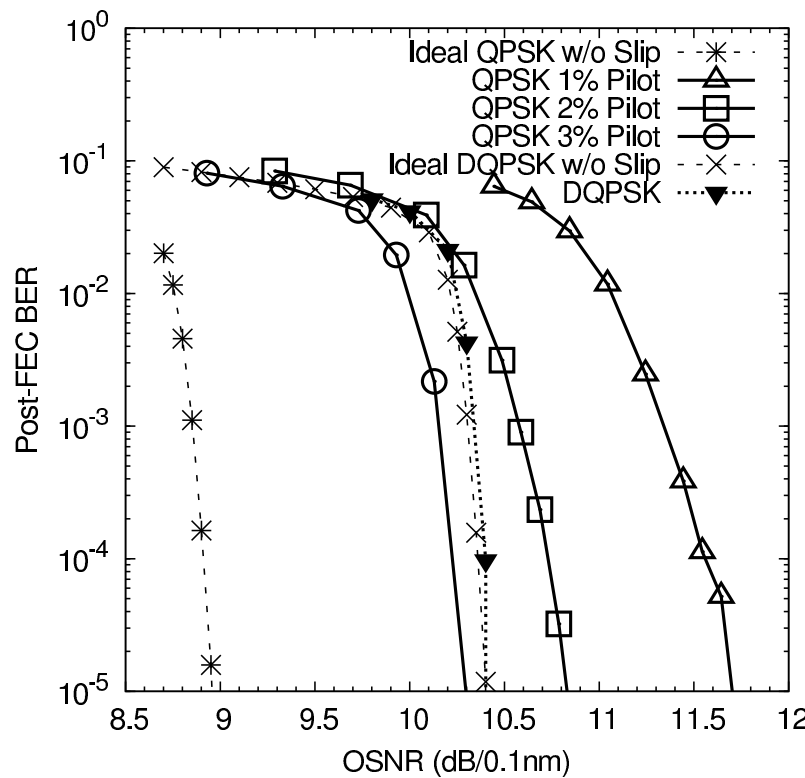

Fig. 3: Post-FEC BER vs. OSNR w/o turbo outer-loop.

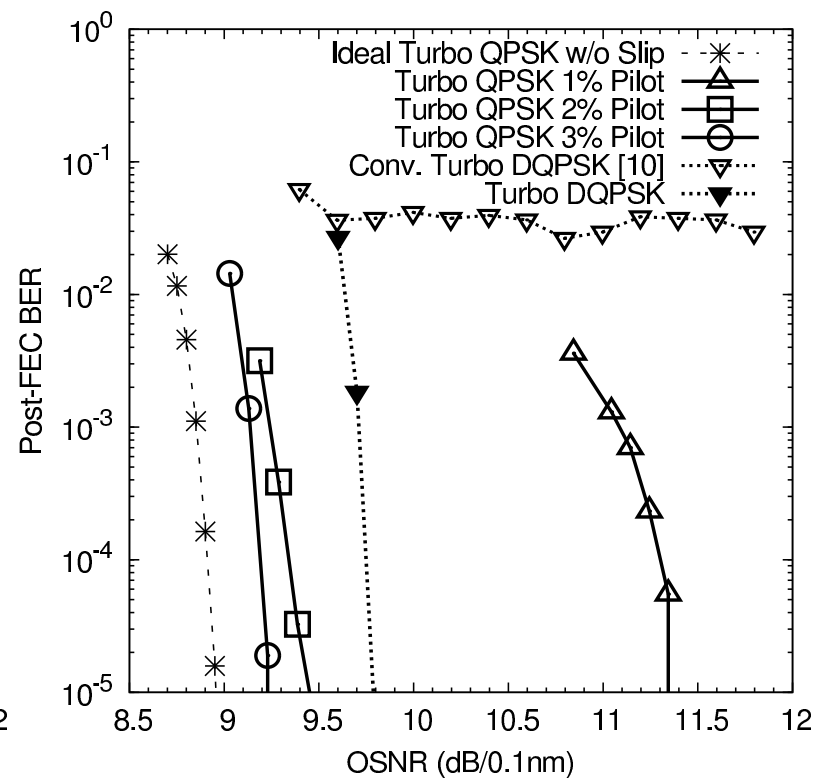

Fig. 4: Post-FEC BER vs. OSNR w/ turbo outer-loop.

From the above results, without a turbo outer-loop, there is no obvious advantage of using pilot-aided QPSK over DQPSK in the presence of cycle slips. However, the situation is different when we employ turbo outer-loop iterations as shown in Fig. 4, where we use just 4 inner-loop iterations for LDPC decoding and 8 outer-loop turbo iterations such that the total complexity becomes comparable to the one in Fig. 3. One can see that the turbo loop offers a significant performance improvement; more specifically, $0.85 \mathrm{~dB}, 1.4 \mathrm{~dB}$, and $1.05 \mathrm{~dB}$ gain for $1 \%, 2 \%$, and $3 \%$ pilot insertion at a BER of $10^{-5}$ in comparison to Fig. 3. For $3 \%$ pilot insertion, the post-FEC BER is only $0.3 \mathrm{~dB}$ away (it includes inherent $0.13 \mathrm{~dB}$ loss caused by $3 \%$ pilots) from the ideal QPSK performance, and it becomes $1.4 \mathrm{~dB}$ better than DQPSK without turbo demodulation. We also compare our Markov-based turbo demodulation with the conventional turbo DQPSK demodulation [10]. It is clearly shown that the conventional turbo DQPSK demodulation does not work well in the presence of cycle slips as in [10] because the demodulator does not consider slip rate properly. On the contrary, our modified turbo DQPSK demodulation performs well with $0.6 \mathrm{~dB}$ gain compared to DQPSK demodulation without turbo loop in Fig. 3, whereas it is still $0.6 \mathrm{~dB}$ worse than the turbo QPSK demodulation with $3 \%$ pilot insertion. Consequently, QPSK can outperform DQPSK with our proposed turbo demodulation even in the presence of frequent cycle slips. QPSK becomes even better with lower-overhead pilots for lower-rate cycle slips.

\section{Conclusions}

We investigated the impact of turbo loop in the post-FEC BER of LDPC-coded QPSK with scattered pilots in the presence of cycle slips. It was shown that QPSK can outperform DQPSK when using our Markov-based turbo demodulation. We can observe the turbo gain of $1.05 \mathrm{~dB}$, which is only $0.3 \mathrm{~dB}$ away from the ideal performance. Note that the required pilot overhead can be lower when we employ more sophisticated CPRs and longer interleaver sizes.

\section{Acknowledgments}

This work was in part supported by the Lambda Reach project of the NICT, as part of a program of the MIC of Japan.

\section{References}

1. A. Bisplinghoff, et al., OFC, OTu3I.1 (2013).

2. M. Taylor, J. Lightwave Technol., 27, 7 (2009).

3. H. Zhang, et al., OFC, OMJ7 (2011).

4. X. Wu, et al., CEC-Net, (2011).

5. I. B. Djordjevic, et al., IEEE JSAC, 26, 6 (2008).

6. C. Duan, et al., OFC, JW2A.5 (2012).

7. T. Fujimori, et al., OECC, (2013).
8. T. Sugihara, et al., OFC, OM2B.3 (2013).

9. K. Sugihara, et al., OFC, OM2B.4 (2013).

10. F. Yu, et al., ECOC, We.10.P1 (2011).

11. A. Bisplinghoff, et al., ECOC, Mo.1.A.5 (2012).

12. Y. Zhao, et al., OFC, OW1H.3 (2012).

13. L. Barletta, et al., J. Lightwave Technol., 25, 13 (2013).

14. T. Yoshida, et al., ECOC, Tu.3.A.1 (2011). 